\title{
On Blockwise Symmetric Matchgate Signatures and Higher Domain \#CSP
}

\author{
Zhiguo $\mathrm{Fu}^{*}$
}

\begin{abstract}
For any $n \geq 3$ and $q \geq 3$, we prove that the EQUALITY function $\left(=_{n}\right)$ on $n$ variables over a domain of size $q$ cannot be realized by matchgates under holographic transformations. This is a consequence of our theorem on the structure of blockwise symmetric matchgate signatures. This has the implication that the standard holographic algorithms based on matchgates, a methodology known to be universal for \#CSP over the Boolean domain, cannot produce P-time algorithms for planar \#CSP over any higher domain $q \geq 3$.
\end{abstract}

${ }^{*}$ School of Mathematics, Jilin University; fuzg@jlu.edu.cn. 


\section{Introduction}

Half a century ago, the Fisher-Kasteleyn-Temperley (FKT) algorithm was discovered [20, 17, 18]. The FKT algorithm can count the number of perfect matchings (dimers) over planar graphs in polynomial time. This is a milestone in the long history in statistical physics and combinatorial algorithms. But the case over general graphs is different. In $1979 \mathrm{~L}$. Valiant [26] defined the class \#P for counting problems. Most counting problems of a combinatorial nature, Sum-of-Product computations such as partition functions studied in physics, and counting Constraint Satisfaction Problems (\#CSP) are all included in \#P (or more precisely in $\mathrm{FP}^{\# \mathrm{P}}$ as the output may be non-integers). In particular, counting perfect matchings in general graphs is \#P-complete [21].

In two seminal papers [22, 25], L. Valiant introduced matchgates and holographic algorithms. Computation in holographic algorithms based on matchgates is expressed and interpreted through a choice of linear basis vectors in an exponential "holographic" mix. Then the actual computation is carried out, via the Holant Theorem, by the FKT algorithm for counting the number of perfect matchings in a planar graph. This methodology has produced polynomial time algorithms for a variety of problems ranging from restrictive versions of Satisfiability, Vertex Cover, to other graph problems such as edge orientation and node/edge deletion. No polynomial time algorithms were known for any of these problems, and some minor variations are known to be NP-hard.

In the past decade significant progress was made in the understanding of these remarkable algorithms [3, 5, 6, 10, 14, 19, 23, 25, 24]. In an interesting twist, it turns out that the idea of a holographic reduction is not only a powerful technique to design unexpected algorithms (tractability), but also an indispensable tool to prove intractability and then to prove classification theorems. Furthermore, in a self-referential twist, it has proved to be a crucial tool to understand the limit and scope of the newly introduced holographic algorithms themselves [9, 13, 16, 15, 5, 14, 3. This study has produced a number of complexity dichotomy theorems. These classify every problem expressible in a framework as either solvable in P-time or being \#P-hard, with nothing in between.

One such framework is called (weighted) \#CSP problems. Let $[q]=\{0,1, \cdots, q-1\}$ denote a domain of size $q$. A \#CSP problem over the domain $[q]$ (for $q=2$, it is the Boolean domain) is specified by a fixed finite set $\mathcal{F}$ of local constraint functions. Each function $f \in \mathcal{F}$ has an arity $k$, and maps $[q]^{k} \rightarrow \mathbb{C}$. (Unweighted \#CSP problems are defined by 0-1 valued constraint functions.) An instance of \# $\operatorname{CSP}(\mathcal{F})$ is specified by a finite set of variables $X=\left\{x_{1}, x_{2}, \ldots, x_{n}\right\}$, and a finite sequence of constraints $\mathcal{S}$ from $\mathcal{F}$, each applied to an ordered sequence of variables from $X$. The output of this instance is $\left.\sum_{\sigma} \prod_{f \in \mathcal{S}} f\right|_{\sigma}$, a sum over all $\sigma: X \rightarrow[q]$, of products of all constraints in $\mathcal{S}$ evaluated according to $\sigma$. \#CSP is a

very expressive framework for locally specified counting problems. E.g., all spin systems are special cases where $\mathcal{F}$ consists of a single binary constraint, and possibly some unary constraints when there are "external fields".

The following classification theorem for \#CSP on the Boolean domain is proved in gradually increasing generalities [10, 14, 2, reaching full generality in [2]:

Theorem 1.1. For any finite set of constraint functions $\mathcal{F}$ over Boolean variables, each taking (algebraic) complex values and not necessarily symmetric, $\# C S P(\mathcal{F})$ belongs to exactly one of three categories according to $\mathcal{F}$ : (1) It is P-time solvable; (2) It is P-time solvable over planar graphs but \#P-hard over general graphs; (3) It is \#P-hard over planar graphs. Moreover, category (2) consists precisely of those problems that are holographically reducible to the FKT algorithm, whereby all constraint functions in $\mathcal{F}$ and EQUALITY functions of all arities are transformed to matchgate signatures.

Theorem 1.1 shows that holographic algorithms with matchgates form a universal strategy, that applies a holographic transformation whereby we transform all EQUALITY functions to matchgates, for all problems in this framework that are \#P-hard in general but solvable in polynomial time on planar 
graphs. But for \#CSP over higher domains, the situation is different. Over the general domain of size $q \geq 3$, there are only a few holographic algorithms with matchgates [27, 1. But it can be argued that they are problems that actually get transformed to a Boolean domain \#CSP problems. In the present paper, we prove the following theorem:

Theorem 1.2. For any $n \geq 3$ and $q \geq 3$, there is no $q \times 2^{\ell}$ matrix $M$ of rank $q$ such that the transformed EQUALITY function $\left(=_{n}\right) M^{\otimes n}$ by $M$ can be realized by a matchgate signature, where $\left(={ }_{n}\right)$ is the EQUALITY function on $n$ variables over the domain $[q]$.

This result has the consequence that the standard strategy that is universal for \#CSP on the Boolean domain, cannot work in any higher domain $[q]$, where $q \geq 3$. In the proof of Theorem [1.2, a main tool is the use of Matchgate Identities (MGI). These are a set of polynomial equations on the entries of a matchgate realizable constraint function (signature) and they have deep relations to some branches of mathematics and complexity theory. In [7, 11, 28], MGI were used to prove the basis collapse theorems and many techniques were developed. In the present paper, we contribute some new techniques to use MGI.

Theorem 1.2 is a part of our effort to achieve a full structural characterization of blockwise symmetric matchgate signatures. This improves upon the work by Cai and $\mathrm{Lu}[8]$. Our characterization theorem will be stated in Theorem 4.1, after we have defined all the necessary terminology. Compared to the theorem in [8] we eliminate a non-vanishing condition and improve the characterization to all arity $n \geq 3$. This improvement makes Theorem 1.2 above possible. We also give a counter example to indicate that Theorem 4.1 cannot be further improved to $n=2$. So we have achieved a complete characterization of the structure of blockwise symmetric matchgate signatures.

Beyond \#CSP, there is a framework called Holant problems for locally specified counting problems, of which \#CSP is a special case. In [3], it is proved that, the analogous universality statement for Holant problems over the Boolean domain is not true: In addition to holographic reductions to matchgates, there is another set of problems (expressible in the Holant framework but not in \#CSP) that is \#P-hard in general but P-time tractable over planar graphs. Moreover they cannot be transformed to matchgates, and the P-time algorithm is not the type discussed in this paper. Thus the present paper also highlights the distinction and importance of Holant problems, beyond \#CSP. It is an open question how to classify Holant problems for higher domains, especially those problems that are \#P-hard in general but P-time tractable over planar graphs.

\section{Preliminaries}

In the following, we always use $e_{i}$ to denote the string with 1 in the $i$-th bit and 0 elsewhere (of some length $n$ )and use $\mathbf{0}$ to denote the $\ell$-bit string $00 \cdots 0$.

Let $G=(V, E, W)$ be a weighted undirected planar graph. A matchgate $\Gamma$ is a tuple $(G, X)$ where $X \subset V$ is a set of external nodes, ordered counterclockwise on the external face. $\Gamma$ is called an odd (resp. even) matchgate if it has an odd (resp. even) number of nodes.

Each matchgate $\Gamma$ with $n$ external nodes is assigned a matchgate signature $\left(\Gamma^{\alpha}\right)_{\alpha \in\{0,1\}^{n}}$ with $2^{n}$ entries,

$$
\Gamma^{i_{1} i_{2} \cdots i_{n}}=\operatorname{PerfMatch}(G-Z)=\sum_{M} \prod_{(i, j) \in M} w_{i j}
$$

where the sum is over all perfect matchings $M$ of $G-Z$, and $Z \subset X$ is a subset of external nodes having the characteristic sequence $\chi_{Z}=i_{1} i_{2} \cdots i_{n}$ and $G-Z$ is obtained from $G$ by removing $Z$ and its incident edges. 
An entry $\Gamma^{\alpha}$ is called an even (resp. odd) entry if the Hamming weight wt $(\alpha)$ is even (resp. odd). It was proved in [4] that matchgate signatures are characterized by the following two sets of conditions. (1) The parity requirements: either all even entries are 0 or all odd entries are 0 . This is due to perfect matchings. (2) A set of Matchgate Identities (MGI) defined as follows: For any $\alpha \in\{0,1\}^{n}$ and any position vector $P=\left\{p_{1}, p_{2}, \cdots, p_{\ell}\right\}$, where $p_{1}<p_{2}<\cdots<p_{\ell}$, also denoted by a bit string,

$$
\sum_{i=1}^{\ell}(-1)^{i} \Gamma^{\alpha+e_{p_{i}}} \Gamma^{\alpha+P+e_{p_{i}}}=0
$$

(alternating sum by flipping in sequence the bits $p_{i}$ and the bits in $P \backslash\left\{p_{i}\right\}$ ), where $\alpha+\beta$ denotes the XOR of $\alpha$ and $\beta$. Actually in [4] it is shown that MGI implies the Parity Condition. But in practice, it is easier to apply the Parity Condition first. We use $\mathscr{M}$ to denote the set of matchgate signatures.

Now we introduce Holant problems over domain $[q]$. Fix a set $\mathcal{F}$ of local constraint functions, a.k.a. signatures. A signature grid $\Omega=(G, \pi)$ consists of a graph $G=(V, E)$, and a mapping $\pi$ which maps each vertex $v \in V$ to some $f_{v} \in \mathcal{F}$ on domain $\{0,1, \cdots, q-1\}$ of arity $\operatorname{deg}(v)$ (= the number of input variables of $f_{v}$ ), and associates its incident edges $E(v)$ to the input variables of $f_{v}$. We say that $\Omega$ is a planar signature grid if $G$ is a plane graph, where the variables of $f_{v}$ are ordered counterclockwise starting from an edge specified by $\pi$. The Holant problem on instance $\Omega$ is to evaluate

$$
\operatorname{Holant}(\Omega ; \mathcal{F})=\sum_{\sigma: E \rightarrow\{0,1, \cdots, q-1\}} \prod_{v \in V} f_{v}\left(\left.\sigma\right|_{E(v)}\right)
$$

where $\left.\sigma\right|_{E(v)}$ denotes the restriction of $\sigma$ to $E(v)$. A Holant problem is parameterized by a set of signatures. Given a set of signatures $\mathcal{F}$, the counting problem Holant $(\mathcal{F})$ is as follows: The input is a signature grid $\Omega=(G, \pi)$; the output is $\operatorname{Holant}(\Omega ; \mathcal{F})$. The problem Pl-Holant $(\mathcal{F})$ is defined similarly using a planar signature grid. For example, for domain size $q=2$, if we place the ExACT-OnE function at every vertex, then Pl-Holant $(\Omega ; \mathcal{F})$ counts the number of PERFECT Matchings. Note that this problem can be computed in polynomial time by the Kasteleyn's algorithm (a.k.a. the FKT algorithm) [17, 18, 20]. Moreover, if $\mathcal{F} \subseteq \mathscr{M}$, then Pl-Holant $(\mathcal{F})$ can be computed in polynomial time. We use $\left(={ }_{n}\right)$ to denote the $n$-ary EQUALITY signature, i.e., the signature takes 1 on the input $x_{1}=x_{2}=\cdots=x_{n}$ and 0 on other inputs.

To define holographic reductions, we use $\operatorname{Holant}(\mathcal{F} \mid \mathcal{G})$ to denote the Holant problem over signature grids with a bipartite graph $G=(U, V, E)$, where each vertex in $U$ or $V$ is assigned a signature in $\mathcal{F}$ or $\mathcal{G}$, respectively. Signatures in $\mathcal{F}$ are given as row vectors listing the function values like truth tables; signatures in $\mathcal{G}$ are given as column vectors. We use Pl-Holant $(\mathcal{F} \mid \mathcal{G})$ to denote the Holant problem over planar bipartite graphs. Counting constraint satisfaction problems (\#CSP) can be defined as a special case of Holant problems. An instance of $\# \operatorname{CSP}(\mathcal{F})$ is presented as a bipartite graph. There is one node for each variable on LHS and for each occurrence of constraint functions on RHS respectively. Connect a constraint node to a variable node if the variable appears in that occurrence of constraint, with a labeling on the edges for the order of these variables. This bipartite graph is also known as the constraint graph. If we attach each variable node with an EQUALITY signature, and consider every edge as a variable, then the \#CSP problem is just the Holant problem on this bipartite graph. Thus $\# \operatorname{CSP}(\mathcal{F}) \equiv_{T} \operatorname{Holant}(\mathcal{E} \mathcal{Q} \mid \mathcal{F})$, where $\mathcal{E} \mathcal{Q}=\left\{=_{1},=_{2},=_{3}, \ldots\right\}$ is the set of EQUALITY signatures of all arities. By restricting to planar constraint graphs, we have Pl-\#CSP.

Now we define holographic transformations. Let $M$ be a $q \times 2^{\ell}$ matrix of rank $q$, then there exists a $2^{\ell} \times q$ matrix $\check{M}$ of rank $q$ such that $M \check{M}=I_{q}$. For a signature $f$ of arity $n$ on domain $\{0,1, \cdots, q-1\}$, written as a column vector $f \in \mathbb{C}^{q^{n}}$, we denote by $\check{M} f=(\check{M})^{\otimes n} f$ the transformed signature. For a signature set $\mathcal{F}$, define $\check{M} \mathcal{F}=\{\check{M} f \mid f \in \mathcal{F}\}$. For signatures written as row vectors we define 
$\mathcal{F} M$ similarly. In particular, for $q=2$, let $H_{2}=\frac{1}{\sqrt{2}}\left[\begin{array}{ll}1 & 1 \\ 1 & -1\end{array}\right]$ be the Hadamard matrix and we denote $\widehat{\mathcal{F}}=H_{2} \mathcal{F}$. Note that $\left(H_{2}\right)^{-1}=H_{2}$ and $\mathcal{E} \mathcal{Q} \subseteq \widehat{\mathscr{M}}$. This fact, that under the Hadamard transformation all EQUALITIES' become matchgate signatures is proved to be the universal reason that \#CSP problems that are \#P-hard can become P-time tractable on planar structures. This is proved in [2].

The holographic transformation defined by $M$ is the following operation: given a signature grid $\Omega=(G, \pi)$ of Holant $(\mathcal{F} \mid \mathcal{G})$, for the same bipartite graph $G$, we get a new signature grid $\Omega^{\prime}=\left(G, \pi^{\prime}\right)$ of Holant $(\mathcal{F} M \mid \check{M} \mathcal{G})$ by replacing each signature in $\mathcal{F}$ or $\mathcal{G}$ with the corresponding signature in $\mathcal{F} M$ or $\check{M} \mathcal{G}$. Then we have the following theorem.

Theorem 2.1. (Valiant's Holant Theorem[25]). Let $\mathcal{F}, \mathcal{G}$ be signature sets on domain $\{0,1, \cdots, q-1\}$ and $M$ be a $q \times 2^{\ell}$ matrix of rank $q$, then

$$
\operatorname{Holant}(\mathcal{F} \mid \mathcal{G})=\operatorname{Holant}(\mathcal{F} M \mid \check{M} \mathcal{G}) .
$$

By Theorem 2.1 and $\mathcal{E Q} \subseteq \widehat{\mathscr{M}}$, if $\mathcal{F} \subseteq \widehat{\mathscr{M}}$, then $\operatorname{Pl}-\# \operatorname{CSP}(\mathcal{F})$ can be computed in polynomial time by the holographic transformation using $H_{2}$ and the FKT algorithm. More precisely, we have the following complexity trichotomy theorem for \#CSP on the Boolean domain [10, 14, 2]: every single problem in this class can be classified into one of three types. The first type can be solved in polynomial time over arbitrary structures. The second type consists of problems that are \#P-hard over general structures, but solvable in polynomial time over planar structures. The third type problems are those which remain \#P-hard over planar structures. In this trichotomy theorem, the second type of problems are precisely captured by $\widehat{\mathscr{M}}$.

Definition 2.1. A signature $f=\left(f^{i_{1} i_{2} \cdots i_{n}}\right)$, where each $i_{j} \in[q]$, is symmetric if $f$ is invariant under any permutation of $\left\{i_{1}, i_{2}, \cdots, i_{n}\right\}$ i.e., $f^{i_{1} i_{2} \cdots i_{n}}=f^{i_{\sigma(1)} i_{\sigma(2)} \cdots i_{\sigma(n)}}$ for any permutation $\sigma$ of $\{1,2 \cdots, n\}$.

For example, the EQUALITY signatures are symmetric.

Definition 2.2. For an $n$-ary symmetric signature $f$ over domain $[q]$, its matrix form $M(f)$ is a $q \times q^{n-1}$ matrix. Its rows are indexed by $i_{1}$ and its columns are indexed by $i_{2} i_{3} \cdots i_{n}$.

For a string $\alpha=\left(i_{1} i_{2} \cdots i_{\ell}\right)\left(i_{\ell+1} i_{\ell+2} \cdots i_{2 \ell}\right) \cdots\left(i_{(n-1) \ell+1} i_{(n-1) \ell+2} \cdots i_{n \ell}\right) \in\{0,1\}^{n \ell}$, we call $\alpha_{j}=$ $i_{(j-1) \ell+1} i_{(j-1) \ell+2} \cdots i_{j \ell}, 1 \leq j \leq n$, blocks in size- $\ell$ and call $\alpha=\alpha_{1} \alpha_{2} \cdots \alpha_{n}$ the blockwise form of $\alpha$ in size- $\ell$.

Definition 2.3. An $n \ell$-ary signature $\Gamma$ over the Boolean domain is blockwise symmetric in size- $\ell$ if $\Gamma=\left(\Gamma^{\alpha_{1} \alpha_{2} \cdots \alpha_{n}}\right)$, where each $\alpha_{i} \in\{0,1\}^{\ell}$, is invariant under any permutation of $\left\{\alpha_{1}, \alpha_{2}, \cdots, \alpha_{n}\right\}$ i.e., $\Gamma^{\alpha_{1} \alpha_{2} \cdots \alpha_{n}}=\Gamma^{\alpha_{\sigma(1)} \alpha_{\sigma(2)} \cdots \alpha_{\sigma(n)}}$ for any permutation $\sigma$ of $\{1,2 \cdots, n\}$. In particular, if $\ell=1$, we say $\Gamma$ is bitwise symmetric.

If it is clear in the context, we will omit size- $\ell$.

Definition 2.4. The matrix form $M(\Gamma)$ of the blockwise symmetric signature $\Gamma=\left(\Gamma^{\alpha_{1} \alpha_{2} \cdots \alpha_{n}}\right)$ of arity $n \ell$ is a $2^{\ell} \times 2^{(n-1) \ell}$ matrix. Its rows are indexed by $\alpha_{1}$ and its columns are indexed by $\alpha_{1} \alpha_{2} \cdots \alpha_{n}$.

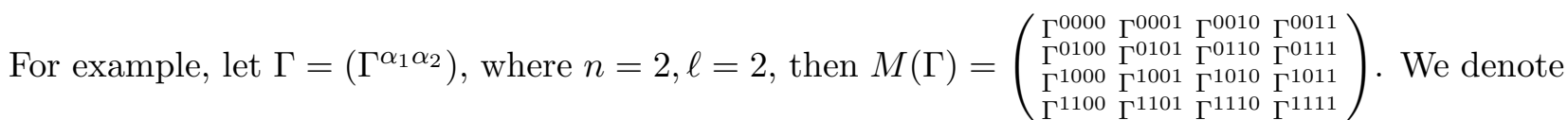
the $\alpha$-th row of $M(\Gamma)$ by $M(\Gamma)^{\alpha}$ for $\alpha \in\{0,1\}^{\ell}$.

The following is a simple lemma from Linear Algebra.

Lemma 2.1. Let $A, B, C$ be $m \times n, n \times s, s \times t$ matrices respectively, where $\operatorname{rank}(A)=n$, $\operatorname{rank}(C)=s$, then $\operatorname{rank}(A B)=\operatorname{rank}(B), \operatorname{rank}(B C)=\operatorname{rank}(B)$. 
Lemma 2.2. Let $f M^{\otimes n}=\Gamma$, where $f$ is an n-ary signature on domain $[q], M$ is a $q \times 2^{\ell}$ matrix and $\Gamma=\left(\Gamma^{\alpha_{1} \alpha_{2} \cdots \alpha_{n}}\right)$ is an $n \ell$-ary signature where $\alpha_{i} \in\{0,1\}^{\ell}$ for $1 \leq i \leq n$. If $f$ is symmetric, then $\Gamma$ is blockwise symmetric.

Proof. Let $M=\left(M_{i}^{\alpha}\right)$, where $i \in[n]$ is the index of rows and $\alpha \in\{0,1\}^{\ell}$ is the index of columns. From $\Gamma=f M^{\otimes n}$, we have $\Gamma^{\alpha_{1} \alpha_{2} \cdots \alpha_{n}}=\sum_{i_{1}, i_{2}, \cdots, i_{n} \in[q]} f^{i_{1} i_{2} \cdots i_{n}} M_{i_{1}}^{\alpha_{1}} M_{i_{2}}^{\alpha_{2}} \cdots M_{i_{n}}^{\alpha_{n}}$. Then for any permutation $\sigma$ of $\{1,2, \cdots, n\}$, we have

$$
\Gamma^{\alpha_{\sigma(1)} \alpha_{\sigma(2)} \cdots \alpha_{\sigma(n)}}=\sum_{i_{\sigma(1)}, i_{\sigma(2)}, \cdots, i_{\sigma(n)} \in[q]} f^{i_{\sigma(1)} i_{\sigma(2)} \cdots i_{\sigma(n)}} M_{i_{\sigma(1)}}^{\alpha_{\sigma(1)}} M_{i_{\sigma(2)}}^{\alpha_{\sigma(2)}} \cdots M_{i_{\sigma(n)}}^{\alpha_{\sigma(n)}} .
$$

Note that $f^{i_{\sigma(1)} i_{\sigma(2)} \cdots i_{\sigma(n)}}=f^{i_{1} i_{2} \cdots i_{n}}$ since $f$ is symmetric, and $M_{i_{1}}^{\alpha_{1}} M_{i_{2}}^{\alpha_{2}} \cdots M_{i_{n}}^{\alpha_{n}}=M_{i_{\sigma(1)}}^{\alpha_{\sigma(1)}} M_{i_{\sigma(2)}}^{\alpha_{\sigma(2)}} \cdots M_{i_{\sigma(n)}}^{\alpha_{\sigma(n)}}$ since $\left\{M_{i_{\sigma(1)}}^{\alpha_{\sigma(1)}}, M_{i_{\sigma(2)}}^{\alpha_{\sigma(2)}}, \cdots, M_{i_{\sigma(n)}}^{\alpha_{\sigma(n)}}\right\}$ is just a permutation of $\left\{M_{i_{1}}^{\alpha_{1}}, M_{i_{2}}^{\alpha_{2}}, \cdots, M_{i_{n}}^{\alpha_{n}}\right\}$. Thus $\Gamma^{\alpha_{1} \alpha_{2} \cdots \alpha_{n}}=$ $\Gamma^{\alpha_{\sigma(1)} \alpha_{\sigma(2)} \cdots \alpha_{\sigma(n)}}$. This implies that $\Gamma$ is blockwise symmetric.

If $f M^{\otimes n}=\Gamma$, where $f$ is an $n$-ary symmetric signature on domain $[q]$ and $M$ is a $q \times 2^{\ell}$ matrix, then $\Gamma=\left(\Gamma^{\alpha_{1} \alpha_{2} \cdots \alpha_{n}}\right)$, where $\alpha_{i} \in\{0,1\}^{\ell}$ for $1 \leq i \leq n$, is a blockwise symmetric signature by Lemma 2.2 . Then we have the following lemma.

Lemma 2.3. (Lemma 2.2 of [1]) $M(\Gamma)=M^{T} M(f) M^{\otimes(n-1)}$, where $M^{T}$ is the transpose of $M$.

\section{Equality $\left(={ }_{n}\right)$ with $n \geq 3$ on domain size $\geq 3$ cannot be realized by matchgates}

Let $\Gamma=\left(\Gamma^{\alpha_{1} \alpha_{2} \cdots \alpha_{n}}\right)$, where $\alpha_{i} \in\{0,1\}^{\ell}$ for $1 \leq i \leq n$, be a blockwise symmetric matchgate signature and $M(\Gamma)$ be the matrix form of $\Gamma$. If $\operatorname{rank}(M(\Gamma)) \geq 2$, then there exist $\sigma, \tau \in\{0,1\}^{\ell}$ that satisfy the following conditions:

- $M(\Gamma)^{\sigma}$ and $M(\Gamma)^{\tau}$ are linearly independent,

- $\operatorname{wt}(\sigma+\tau)=\min _{u, v \in\{0,1\}^{\ell}}\left\{\operatorname{wt}(u+v) \mid M(\Gamma)^{u}\right.$ and $M(\Gamma)^{v}$ are linearly independent $\}$,

where $\sigma+\tau$ is the XOR of the bit strings $\sigma$ and $\tau$. Moreover, For any $\beta=\alpha_{1} \cdots \alpha_{t-1} \alpha_{t+1} \cdots \alpha_{n} \in$ $\{0,1\}^{(n-1) \ell}$, let $x_{\beta}=\left(\begin{array}{l}\Gamma^{\alpha_{1} \cdots \alpha_{t-1} \sigma \alpha_{t+1} \cdots \alpha_{n}} \\ \Gamma^{\alpha_{1} \cdots \alpha_{t-1} \tau \alpha_{t+1} \cdots \alpha_{n}}\end{array}\right)$. Then there exist $\zeta, \eta$ satisfy the following conditions:

- $x_{\zeta}$ and $x_{\eta}$ are linearly independent,

- $\operatorname{wt}(\zeta+\eta)=\min _{u, v \in\{0,1\}^{(n-1) \ell}}\left\{\operatorname{wt}(u+v) \mid x_{u}\right.$ and $x_{v}$ are linearly independent $\}$.

For such $\sigma, \tau, \zeta, \eta$, the following lemma is given in [1], which uses the properties of Matchgate (MGI).

Lemma 3.1. If $\operatorname{rank}(M(\Gamma)) \geq 2$, then $\operatorname{wt}(\sigma+\tau)=1$ and $\operatorname{wt}(\zeta+\eta)=1$.

For a blockwise symmetric matchgate signature $\Gamma=\left(\Gamma^{\alpha_{1} \alpha_{2} \cdots \alpha_{n}}\right)$ of arity $n \ell$, by Lemma 3.1 and the parity condition, if $\operatorname{rank}(M(\Gamma)) \geq 2$, then $M(\Gamma)$ has a full rank submatrix of the form (zeros are due to Parity Constraint)




where $e_{s}, e_{t} \in\{0,1\}^{\ell}$.

Moreover, let $\Gamma=\left(\Gamma^{\alpha_{1} \alpha_{2} \cdots \alpha_{n}}\right)$ be a blockwise symmetric matchgate signature of arity $n \ell$. If $\operatorname{rank}(M(\Gamma)) \geq 3$, then there exist $\sigma, \tau \in\{0,1\}^{\ell}$ satisfy the following conditions:

- $\operatorname{wt}(\sigma)$ and $\operatorname{wt}(\tau)$ have the same parity,

- $M(\Gamma)^{\sigma}$ and $M(\Gamma)^{\tau}$ are linearly independent,

- $\operatorname{wt}(\sigma+\tau)=\min _{u, v \in\{0,1\}^{\ell}}\left\{\operatorname{wt}(u+v) \mid \operatorname{wt}(u)\right.$ and $\operatorname{wt}(v)$ have the same parity and $M(\Gamma)^{u}, M(\Gamma)^{v}$ are linearly independent $\}$.

Moreover, For any $\beta=\alpha_{1} \cdots \alpha_{t-1} \alpha_{t+1} \cdots \alpha_{n} \in\{0,1\}^{(n-1) \ell}$, let $x_{\beta}=\left(\begin{array}{l}\Gamma^{\alpha_{1} \cdots \alpha_{t-1} \sigma \alpha_{t+1} \cdots \alpha_{n}} \\ \Gamma^{\alpha_{1} \cdots \alpha_{t-1} \tau \alpha_{t+1} \cdots \alpha_{n}}\end{array}\right)$. Then there exist $\zeta, \eta$ satisfy the following condition:

- $x_{\zeta}$ and $x_{\eta}$ are linearly independent,

- $\operatorname{wt}(\zeta+\eta)=\min _{u, v \in\{0,1\}^{(n-1) \ell}}\left\{\operatorname{wt}(u+v) \mid x_{u}\right.$ and $x_{v}$ are linearly independent $\}$.

For such $\sigma, \tau, \zeta, \eta$, the following Lemma is given in [1] (again using MGI),

Lemma 3.2. If $\operatorname{rank}(M(\Gamma)) \geq 3$, then $\operatorname{wt}(\sigma+\tau)=2, \operatorname{wt}(\zeta+\eta)=2$.

For a blockwise symmetric matchgate signature $\Gamma=\left(\Gamma^{\alpha_{1} \alpha_{2} \cdots \alpha_{n}}\right)$ of arity $n \ell$, Lemma 3.2 implies that if $\operatorname{rank}(M(\Gamma)) \geq 3$, then $M(\Gamma)$ has a full rank submatrix of the form

$$
\left(\begin{array}{cc}
\Gamma_{1}^{\alpha_{1} \alpha_{2} \alpha_{3} \cdots \alpha_{n}} & \Gamma^{\alpha_{1}\left(\alpha_{2}+e_{s}+e_{t}\right) \alpha_{3} \cdots \alpha_{n}} \\
\Gamma^{\left(\alpha_{1}+e_{i}+e_{j}\right) \alpha_{2} \alpha_{3} \cdots \alpha_{n}} & \Gamma^{\left(\alpha_{1}+e_{i}+e_{j}\right)\left(\alpha_{2}+e_{s}+e_{t}\right) \alpha_{3} \cdots \alpha_{n}}
\end{array}\right)
$$

or

$$
\left(\begin{array}{cc}
\Gamma^{\alpha_{1} \alpha_{2} \alpha_{3} \cdots \alpha_{n}} & \Gamma^{\alpha_{1}\left(\alpha_{2}+e_{s}\right)\left(\alpha_{3}+e_{t}\right) \cdots \alpha_{n}} \\
\Gamma^{\left(\alpha_{1}+e_{i}+e_{j}\right) \alpha_{2} \alpha_{3} \cdots \alpha_{n}} & \Gamma^{\left(\alpha_{1}+e_{i}+e_{j}\right)\left(\alpha_{2}+e_{s}\right)\left(\alpha_{3}+e_{t}\right) \cdots \alpha_{n}}
\end{array}\right),
$$

where $e_{i}, e_{j}, e_{s}, e_{t}$ are in $\{0,1\}^{\ell}, s<t$ in (3.1) and $i<j$. But the next two lemmas show that for any $\left\{\beta_{1}, \beta_{2}, \cdots, \beta_{n}, \gamma_{1}, \gamma_{2}, \cdots, \gamma_{n}\right\}$, where $\beta_{i}, \gamma_{i} \in\{0,1\}^{\ell}$, such that $\operatorname{wt}\left(\beta_{1}+\gamma_{1}\right)=2$, wt $\left(\beta_{2} \beta_{3} \cdots \beta_{n}+\right.$ $\left.\gamma_{2} \gamma_{3} \cdots \gamma_{n}\right)=2$, the submatrix of $M(\Gamma)\left(\begin{array}{c}\Gamma^{\beta_{1} \beta_{2} \beta_{3} \cdots \beta_{n}} \\ \Gamma^{\gamma_{1} \beta_{2} \beta_{3} \cdots \beta_{n}} \Gamma^{\beta_{1} \gamma_{2} \gamma_{3} \cdots \gamma_{n}} \\ \Gamma_{3} \gamma_{3} \cdots \gamma_{n}\end{array}\right)$ has rank less than 2 .

Lemma 3.3. Let $\Gamma=\left(\Gamma^{\alpha_{1} \alpha_{2} \cdots \alpha_{n}}\right)$ be a blockwise symmetric matchgate signature of arity $n \ell$, where $n \geq 3$. Then for any $\alpha_{1} \alpha_{2} \cdots \alpha_{n} \in\{0,1\}^{n \ell}$,

$$
A=\left(\begin{array}{cc}
\Gamma^{\alpha_{1} \alpha_{2} \alpha_{3} \cdots \alpha_{n}} & \Gamma^{\alpha_{1}\left(\alpha_{2}+e_{s}\right)\left(\alpha_{3}+e_{t}\right) \cdots \alpha_{n}} \\
\Gamma^{\left(\alpha_{1}+e_{i}+e_{j}\right) \alpha_{2} \alpha_{3} \cdots \alpha_{n}} & \Gamma^{\left(\alpha_{1}+e_{i}+e_{j}\right)\left(\alpha_{2}+e_{s}\right)\left(\alpha_{3}+e_{t}\right) \cdots \alpha_{n}}
\end{array}\right)
$$

is degenerate, where $e_{i}, e_{j}, e_{s}, e_{t} \in\{0,1\}^{\ell}$ and $i<j$.

Proof. We will prove that $\operatorname{det}(A)=0$ by applying Matchgate Identities (MGI).

Let the pattern be $\left(\alpha_{1}+e_{i}\right) \alpha_{2} \alpha_{3} \cdots \alpha_{n}$ and the position vector be $\left(e_{i}+e_{j}\right)\left(e_{s}\right)\left(e_{t}\right) \mathbf{0} \cdots \mathbf{0}$. Then by MGI we have

$$
\begin{aligned}
& \Gamma^{\alpha_{1} \alpha_{2} \alpha_{3} \cdots \alpha_{n}} \Gamma^{\left(\alpha_{1}+e_{i}+e_{j}\right)\left(\alpha_{2}+e_{s}\right)\left(\alpha_{3}+e_{t}\right) \cdots \alpha_{n}}-\Gamma^{\left(\alpha_{1}+e_{i}+e_{j}\right) \alpha_{2} \alpha_{3} \cdots \alpha_{n}} \Gamma^{\alpha_{1}\left(\alpha_{2}+e_{s}\right)\left(\alpha_{3}+e_{t}\right) \cdots \alpha_{n}} \\
+ & \Gamma^{\left(\alpha_{1}+e_{i}\right)\left(\alpha_{2}+e_{s}\right) \alpha_{3} \cdots \alpha_{n}} \Gamma^{\left(\alpha_{1}+e_{j}\right) \alpha_{2}\left(\alpha_{3}+e_{t}\right) \cdots \alpha_{n}}-\Gamma^{\left(\alpha_{1}+e_{i}\right) \alpha_{2}\left(\alpha_{3}+e_{t}\right) \cdots \alpha_{n}} \Gamma^{\left(\alpha_{1}+e_{j}\right)\left(\alpha_{2}+e_{s}\right) \alpha_{3} \cdots \alpha_{n}}=0 .
\end{aligned}
$$

This instantiation of MGI has 4 terms, each component to flipping the bits at position $i, j, s, t$ respectively. 
Moreover, let the pattern be $\left(\alpha_{1}+e_{i}\right) \alpha_{3} \alpha_{2} \alpha_{4} \cdots \alpha_{n}$ and the position vector be $\left(e_{i}+e_{j}\right)\left(e_{t}\right)\left(e_{s}\right) \mathbf{0} \cdots \mathbf{0}$. Then we have

$$
\begin{aligned}
& \Gamma^{\alpha_{1} \alpha_{3} \alpha_{2} \cdots \alpha_{n}} \Gamma^{\left(\alpha_{1}+e_{i}+e_{j}\right)\left(\alpha_{3}+e_{t}\right)\left(\alpha_{2}+e_{s}\right) \cdots \alpha_{n}}-\Gamma^{\left(\alpha_{1}+e_{i}+e_{j}\right) \alpha_{3} \alpha_{2} \cdots \alpha_{n}} \Gamma^{\alpha_{1}\left(\alpha_{3}+e_{t}\right)\left(\alpha_{2}+e_{s}\right) \cdots \alpha_{n}} \\
+ & \Gamma^{\left(\alpha_{1}+e_{i}\right)\left(\alpha_{3}+e_{t}\right) \alpha_{2} \cdots \alpha_{n}} \Gamma^{\left(\alpha_{1}+e_{j}\right) \alpha_{3}\left(\alpha_{2}+e_{s}\right) \cdots \alpha_{n}}-\Gamma^{\left(\alpha_{1}+e_{i}\right) \alpha_{3}\left(\alpha_{2}+e_{s}\right) \cdots \alpha_{n}} \Gamma^{\left(\alpha_{1}+e_{j}\right)\left(\alpha_{3}+e_{t}\right) \alpha_{2} \cdots \alpha_{n}}=0 .
\end{aligned}
$$

Since $\Gamma$ is blockwise symmetric, (3.4) can be rewritten as the following form:

$$
\begin{aligned}
& \Gamma^{\alpha_{1} \alpha_{2} \alpha_{3} \cdots \alpha_{n}} \Gamma^{\left(\alpha_{1}+e_{i}+e_{j}\right)\left(\alpha_{2}+e_{s}\right)\left(\alpha_{3}+e_{t}\right) \cdots \alpha_{n}}-\Gamma^{\left(\alpha_{1}+e_{i}+e_{j}\right) \alpha_{2} \alpha_{3} \cdots \alpha_{n}} \Gamma^{\alpha_{1}\left(\alpha_{2}+e_{s}\right)\left(\alpha_{3}+e_{t}\right) \cdots \alpha_{n}} \\
+ & \Gamma^{\left(\alpha_{1}+e_{i}\right) \alpha_{2}\left(\alpha_{3}+e_{t}\right) \cdots \alpha_{n}} \Gamma^{\left(\alpha_{1}+e_{j}\right)\left(\alpha_{2}+e_{s}\right) \alpha_{3} \cdots \alpha_{n}}-\Gamma^{\left(\alpha_{1}+e_{i}\right)\left(\alpha_{2}+e_{s}\right) \alpha_{3} \cdots \alpha_{n}} \Gamma^{\left(\alpha_{1}+e_{j}\right) \alpha_{2}\left(\alpha_{3}+e_{t}\right) \cdots \alpha_{n}}=0 .
\end{aligned}
$$

Note that the 1,2-th terms of (3.3) are equal to the 1,2-th terms of (3.5) respectively. But the 3 -th term of (3.3) is equal to the 4 -th term of (3.5) and the 4-th term of (3.3) is equal to the 3-th term of (3.5). Thus by adding (3.3) to (3.5), the 3,4-th terms cancel and we have

$$
\Gamma^{\alpha_{1} \alpha_{2} \alpha_{3} \cdots \alpha_{n}} \Gamma^{\left(\alpha_{1}+e_{i}+e_{j}\right)\left(\alpha_{2}+e_{s}\right)\left(\alpha_{3}+e_{t}\right) \cdots \alpha_{n}}-\Gamma^{\left(\alpha_{1}+e_{i}+e_{j}\right) \alpha_{2} \alpha_{3} \cdots \alpha_{n}} \Gamma^{\alpha_{1}\left(\alpha_{2}+e_{s}\right)\left(\alpha_{3}+e_{t}\right) \cdots \alpha_{n}}=0 .
$$

(3.6) implies that the determinant of the matrix $\left(\begin{array}{c}\Gamma^{\alpha_{1} \alpha_{2} \alpha_{3} \cdots \alpha_{n}} \\ \Gamma^{\left(\alpha_{1}+e_{i}+e_{j}\right) \alpha_{2} \alpha_{3} \cdots \alpha_{n}} \\ \Gamma^{\left(\alpha_{1}+e_{i}+e_{j}\right)\left(\alpha_{2}+e_{s}\right)\left(\alpha_{3}+e_{t}\right) \cdots \alpha_{n}}\end{array}\right)$ is zero. This finishes the proof.

By (3.6), we have the following corollary.

Corollary 3.1. For a blockwise symmetric matchgate signature $\Gamma=\left(\Gamma^{\alpha_{1} \alpha_{2} \cdots \alpha_{n}}\right)$ and any $\alpha_{1} \alpha_{2} \cdots \alpha_{n} \in$ $\{0,1\}^{n \ell}$ and $1 \leq i<j \leq \ell, 1 \leq s, t \leq \ell$,

$$
\left(\begin{array}{c}
\Gamma^{\alpha_{1} \alpha_{2} \alpha_{3} \cdots \alpha_{n}} \\
\Gamma^{\left(\alpha_{1}+e_{i}+e_{j}\right) \alpha_{2} \alpha_{3} \cdots \alpha_{n}}
\end{array}\right) \text { and }\left(\begin{array}{c}
\Gamma^{\alpha_{1}\left(\alpha_{2}+e_{s}\right)\left(\alpha_{3}+e_{t}\right) \cdots \alpha_{n}} \\
\Gamma^{\left(\alpha_{1}+e_{i}+e_{j}\right)\left(\alpha_{2}+e_{s}\right)\left(\alpha_{3}+e_{t}\right) \cdots \alpha_{n}}
\end{array}\right)
$$

are linearly dependent.

Lemma 3.4. Let $\Gamma=\left(\Gamma^{\alpha_{1} \alpha_{2} \cdots \alpha_{n}}\right)$ be a blockwise symmetric matchgate signature of arity ne, where $n \geq 3$. Then for any $\alpha_{1} \alpha_{2} \cdots \alpha_{n} \in\{0,1\}^{n \ell}$,

$$
B=\left(\begin{array}{cc}
\Gamma^{\alpha_{1} \alpha_{2} \alpha_{3} \cdots \alpha_{n}} & \Gamma^{\alpha_{1}\left(\alpha_{2}+e_{s}+e_{t}\right) \alpha_{3} \cdots \alpha_{n}} \\
\Gamma^{\left(\alpha_{1}+e_{i}+e_{j}\right) \alpha_{2} \alpha_{3} \cdots \alpha_{n}} & \Gamma^{\left(\alpha_{1}+e_{i}+e_{j}\right)\left(\alpha_{2}+e_{s}+e_{t}\right) \alpha_{3} \cdots \alpha_{n}}
\end{array}\right),
$$

is degenerate, where $e_{i}, e_{j}, e_{s}, e_{t} \in\{0,1\}^{\ell}$ and $i<j$ and $s<t$.

Proof. We will prove that $\operatorname{det}(B)=0$ by applying MGI.

Assume that $\operatorname{det}(B) \neq 0$, firstly, we claim that

$$
\Gamma^{\left(\alpha_{1}+e_{j}\right)\left(\alpha_{3}+e_{u}\right)\left(\alpha_{2}+e_{s}+e_{t}\right) \cdots \alpha_{n}}=\Gamma^{\left(\alpha_{1}+e_{i}\right) \alpha_{2}\left(\alpha_{3}+e_{v}\right) \cdots \alpha_{n}}=0
$$

for any $1 \leq u, v \leq \ell$. If there is $u$ such that $\Gamma^{\left(\alpha_{1}+e_{j}\right)\left(\alpha_{3}+e_{u}\right)\left(\alpha_{2}+e_{s}+e_{t}\right) \cdots \alpha_{n}} \neq 0$, then $\Gamma^{\left(\alpha_{2}+e_{s}+e_{t}\right)\left(\alpha_{1}+e_{j}\right)\left(\alpha_{3}+e_{u}\right) \cdots \alpha_{n}} \neq$ 0 since $\Gamma$ us blockwise symmetric. Thus

$$
\left(\begin{array}{c}
\Gamma^{\alpha_{2}\left(\alpha_{1}+e_{j}\right)\left(\alpha_{3}+e_{u}\right) \cdots \alpha_{n}} \\
\Gamma^{\left(\alpha_{2}+e_{s}+e_{t}\right)\left(\alpha_{1}+e_{j}\right)\left(\alpha_{3}+e_{u}\right) \cdots \alpha_{n}}
\end{array}\right)
$$

is not a zero vector. Since

$$
\begin{gathered}
\left(\begin{array}{c}
\Gamma^{\alpha_{2} \alpha_{1} \alpha_{3} \cdots \alpha_{n}} \\
\Gamma^{\left(\alpha_{2}+e_{s}+e_{t}\right) \alpha_{1} \alpha_{3} \cdots \alpha_{n}}
\end{array}\right) \text { and }\left(\begin{array}{c}
\Gamma^{\alpha_{2}\left(\alpha_{1}+e_{j}\right)\left(\alpha_{3}+e_{u}\right) \cdots \alpha_{n}} \\
\Gamma^{\left(\alpha_{2}+e_{s}+e_{t}\right)\left(\alpha_{1}+e_{j}\right)\left(\alpha_{3}+e_{u}\right) \cdots \alpha_{n}}
\end{array}\right) \\
\left(\begin{array}{c}
\Gamma^{\alpha_{2}\left(\alpha_{1}+e_{i}+e_{j}\right) \alpha_{3} \cdots \alpha_{n}} \\
\Gamma^{\left(\alpha_{2}+e_{s}+e_{t}\right)\left(\alpha_{1}+e_{i}+e_{j}\right) \alpha_{3} \cdots \alpha_{n}}
\end{array}\right) \text { and }\left(\begin{array}{c}
\Gamma^{\alpha_{2}\left(\alpha_{1}+e_{j}\right)\left(\alpha_{3}+e_{u}\right) \cdots \alpha_{n}} \\
\Gamma^{\left(\alpha_{2}+e_{s}+e_{t}\right)\left(\alpha_{1}+e_{j}\right)\left(\alpha_{3}+e_{u}\right) \cdots \alpha_{n}}
\end{array}\right)
\end{gathered}
$$


are linearly dependent respectively by Corollary 3.1 and the nonzero vector in (3.7) appears as the second vector in both cases. Thus

$$
\left(\begin{array}{c}
\Gamma_{2}^{\alpha_{2} \alpha_{1} \alpha_{3} \cdots \alpha_{n}} \\
\Gamma^{\left(\alpha_{2}+e_{s}+e_{t}\right) \alpha_{1} \alpha_{3} \cdots \alpha_{n}}
\end{array}\right) \text { and }\left(\begin{array}{c}
\Gamma^{\alpha_{2}\left(\alpha_{1}+e_{i}+e_{j}\right) \alpha_{3} \cdots \alpha_{n}} \\
\Gamma^{\left(\alpha_{2}+e_{s}+e_{t}\right)\left(\alpha_{1}+e_{i}+e_{j}\right) \alpha_{3} \cdots \alpha_{n}}
\end{array}\right)
$$

are linearly dependent. This contradicts that $\operatorname{det}(B) \neq 0$. Thus $\Gamma^{\left(\alpha_{1}+e_{j}\right)\left(\alpha_{3}+e_{u}\right)\left(\alpha_{2}+e_{s}+e_{t}\right) \cdots \alpha_{n}}=0$ for any $1 \leq u \leq \ell$. By replacing $\alpha_{2}+e_{s}+e_{t}$ with $\alpha_{2}$, replacing $\alpha_{1}+e_{j}$ with $\alpha_{1}+e_{i}$ and replacing $\alpha_{3}+e_{u}$ with $\alpha_{3}+e_{v}$ respectively, we can prove that $\Gamma^{\left(\alpha_{1}+e_{i}\right)\left(\alpha_{3}+e_{v}\right) \alpha_{2} \cdots \alpha_{n}}=0$ for any $v \in[\ell]$ in the same way. Thus $\Gamma^{\left(\alpha_{1}+e_{i}\right) \alpha_{2}\left(\alpha_{3}+e_{v}\right) \cdots \alpha_{n}}=0$ for any $1 \leq v \leq \ell$, i.e., we have

$$
\Gamma^{\left(\alpha_{1}+e_{j}\right)\left(\alpha_{3}+e_{u}\right)\left(\alpha_{2}+e_{s}+e_{t}\right) \cdots \alpha_{n}}=\Gamma^{\left(\alpha_{1}+e_{i}\right) \alpha_{2}\left(\alpha_{3}+e_{v}\right) \cdots \alpha_{n}}=0
$$

for any $1 \leq u, v \leq \ell$.

Now we apply MGI again. Let the pattern be $\left(\alpha_{1}+e_{i}\right) \alpha_{2} \alpha_{3} \alpha_{4} \cdots \alpha_{n}$ and the position vector be $\left(e_{i}+e_{j}\right)\left(\alpha_{2}+\alpha_{3}\right)\left(\alpha_{2}+\alpha_{3}+e_{s}+e_{t}\right) \mathbf{0} \cdots \mathbf{0}$ and $S=\left\{k \mid\right.$ the $k$-th bit of $\alpha_{2}+\alpha_{3}$ is 1$\}, T=\{k \mid$ the $k$-th bit of $\alpha_{2}+\alpha_{3}+e_{s}+e_{t}$ is 1$\}$, then by MGI we have

$$
\begin{aligned}
& \Gamma^{\alpha_{1} \alpha_{2} \alpha_{3} \cdots \alpha_{n}} \Gamma^{\left(\alpha_{1}+e_{i}+e_{j}\right) \alpha_{3}\left(\alpha_{2}+e_{s}+e_{t}\right) \cdots \alpha_{n}}-\Gamma^{\left(\alpha_{1}+e_{i}+e_{j}\right) \alpha_{2} \alpha_{3} \cdots \alpha_{n}} \Gamma^{\alpha_{1} \alpha_{3}\left(\alpha_{2}+e_{s}+e_{t}\right) \cdots \alpha_{n}} \\
& +\sum_{u \in S}\left( \pm \Gamma^{\left(\alpha_{1}+e_{i}\right)\left(\alpha_{2}+e_{u}\right) \alpha_{3} \cdots \alpha_{n}} \Gamma^{\left(\alpha_{1}+e_{j}\right)\left(\alpha_{3}+e_{u}\right)\left(\alpha_{2}+e_{s}+e_{t}\right) \cdots \alpha_{n}}\right) \\
& +\sum_{v \in T}\left( \pm \Gamma^{\left(\alpha_{1}+e_{i}\right) \alpha_{2}\left(\alpha_{3}+e_{v}\right) \cdots \alpha_{n}} \Gamma^{\left(\alpha_{1}+e_{j}\right) \alpha_{3}\left(\alpha_{2}+e_{s}+e_{t}+e_{v}\right) \cdots \alpha_{n}}\right)=0 .
\end{aligned}
$$

Since $\Gamma^{\left(\alpha_{1}+e_{j}\right)\left(\alpha_{3}+e_{u}\right)\left(\alpha_{2}+e_{s}+e_{t}\right) \cdots \alpha_{n}}=\Gamma^{\left(\alpha_{1}+e_{i}\right) \alpha_{2}\left(\alpha_{3}+e_{v}\right) \cdots \alpha_{n}}=0$ for all $1 \leq u, v \leq \ell$, we have

$$
\Gamma^{\alpha_{1} \alpha_{2} \alpha_{3} \cdots \alpha_{n}} \Gamma^{\left(\alpha_{1}+e_{i}+e_{j}\right) \alpha_{3}\left(\alpha_{2}+e_{s}+e_{t}\right) \cdots \alpha_{n}}-\Gamma^{\left(\alpha_{1}+e_{i}+e_{j}\right) \alpha_{2} \alpha_{3} \cdots \alpha_{n}} \Gamma^{\alpha_{1} \alpha_{3}\left(\alpha_{2}+e_{s}+e_{t}\right) \cdots \alpha_{n}}=0
$$

by (3.8). Since $\Gamma$ is blockwise symmetric, we have

$$
\Gamma^{\alpha_{1} \alpha_{2} \alpha_{3} \cdots \alpha_{n}} \Gamma^{\left(\alpha_{1}+e_{i}+e_{j}\right) \alpha_{3}\left(\alpha_{2}+e_{s}+e_{t}\right) \cdots \alpha_{n}}-\Gamma^{\left(\alpha_{1}+e_{i}+e_{j}\right) \alpha_{2} \alpha_{3} \cdots \alpha_{n}} \Gamma^{\alpha_{1} \alpha_{3}\left(\alpha_{2}+e_{s}+e_{t}\right) \cdots \alpha_{n}}=0
$$

by (3.9). Thus we have $\operatorname{det}(B)=0$ and finishes the proof.

Theorem 3.1. If $\Gamma=\left(\Gamma^{\alpha_{1} \alpha_{2} \cdots \alpha_{n}}\right)$ is a blockwise symmetric matchgate signature of arity ne, where $n \geq 3$, then $\operatorname{rank}(M(\Gamma)) \leq 2$.

Proof. If $\operatorname{rank}(M(\Gamma)) \geq 3$, by Lemma 3.2, then there is a full rank submatrix of $M(\Gamma)$ of the following form

or

$$
A=\left(\begin{array}{cc}
\Gamma^{\alpha_{1} \alpha_{2} \alpha_{3} \cdots \alpha_{n}} & \Gamma^{\alpha_{1}\left(\alpha_{2}+e_{s}\right)\left(\alpha_{3}+e_{t}\right) \cdots \alpha_{n}} \\
\Gamma^{\left(\alpha_{1}+e_{i}+e_{j}\right) \alpha_{2} \alpha_{3} \cdots \alpha_{n}} & \Gamma^{\left(\alpha_{1}+e_{i}+e_{j}\right)\left(\alpha_{2}+e_{s}\right)\left(\alpha_{3}+e_{t}\right) \cdots \alpha_{n}}
\end{array}\right)
$$

$$
B=\left(\begin{array}{cc}
\Gamma^{\alpha_{1} \alpha_{2} \alpha_{3} \cdots \alpha_{n}} & \Gamma^{\alpha_{1}\left(\alpha_{2}+e_{s}+e_{t}\right) \alpha_{3} \cdots \alpha_{n}} \\
\Gamma^{\left(\alpha_{1}+e_{i}+e_{j}\right) \alpha_{2} \alpha_{3} \cdots \alpha_{n}} & \Gamma^{\left(\alpha_{1}+e_{i}+e_{j}\right)\left(\alpha_{2}+e_{s}+e_{t}\right) \alpha_{3} \cdots \alpha_{n}}
\end{array}\right),
$$

where $e_{i}, e_{j}, e_{s}, e_{t} \in\{0,1\}^{\ell}$ and $i<j$ and $s<t$. But by Lemma 3.3 and Lemma 3.4, we know both $A$ and $B$ are degenerate. This is a contradiction.

Now we can prove Theorem 1.2 .

Proof. Let $M\left(=_{n}\right)$ be the matrix form of the EQUALITY signature $\left(=_{n}\right)$. Then $M\left(=_{n}\right)_{i, i \cdots i}=1$ for $0 \leq i \leq q-1$ and all other entries of $M\left(=_{n}\right)$ are zero. Thus $\operatorname{rank}\left(M\left(=_{n}\right)\right)=q$. For any $q \times 2^{\ell}$ matrix with rank $q$, let $\Gamma=\left(={ }_{n}\right) M^{\otimes n}$, then $\Gamma$ is blockwise symmetric by Lemma 2.2 and $M(\Gamma)=M^{T}\left(M\left(={ }_{n}\right) M^{\otimes n-1}\right.$ by Lemma 2.3. Since $\operatorname{rank}\left(M\left(=_{n}\right)\right)=q \geq 3, \operatorname{rank}\left(M^{T}\right)=q$ and $\operatorname{rank}\left(M^{\otimes n-1}\right)=q^{(n-1)}$. We have $\operatorname{rank}(M(\Gamma)) \geq 3$ by Lemma 2.1. If $\Gamma$ is realized by a matchgate, i.e., $\Gamma$ is a matchgate signature, then by Theorem $3.1, \operatorname{rank}(M(\Gamma)) \leq 2$. This is a contradiction. 
We remark that the condition $n \geq 3$ is necessary in Theorem 3.1. For example, let $\Gamma=\left(\Gamma^{\alpha_{1} \alpha_{2}}\right)$, where $\alpha_{1}, \alpha_{2} \in\{0,1\}^{2}$, with $\Gamma^{0000}=\Gamma^{1001}=\Gamma^{0110}=1, \Gamma^{1111}=-1$ and all other entries are zero. Note that $\Gamma$ is blockwise symmetric and is realized by the matchgate $\Gamma_{1}$. But $\operatorname{rank}(M(\Gamma))=4$.



$\Gamma_{1}$ : The four nodes at the corner are external nodes and the other two nodes are internal nodes.

The middle edge has weight -1 and all other edges have weight 1 .

Theorem 1.2 implies that the EQUALITIES cannot be realized by matchgates under holographic transformation. Thus we have the following theorem.

Theorem 3.2. For any $q \geq 3$, there is no polynomial time algorithms for $\mathrm{Pl}$ - $\# \operatorname{CSP}(\mathcal{F})(\operatorname{Pl}-\operatorname{Holant}(\mathcal{E} \mathcal{Q} \mid \mathcal{F}))$, for any $\mathcal{F}$ such that $\# \operatorname{CSP}(\mathcal{F})$ is \#P-hard, that is obtained by a holographic transformation $M \in \mathbb{C}^{q \times 2^{\ell}}$ of rank $q$, such that all EQUALITIES in $\mathcal{E} \mathcal{Q}$ are transformed to $\mathscr{M}$.

\section{The structure of blockwise symmetric matchagate signatures}

For an $n$-bit string $\alpha \in\{0,1\}^{n}$, we define $p(\alpha)=0$ if $\operatorname{wt}(\alpha)$ is even and $p(\alpha)=1$ if $\operatorname{wt}(\alpha)$ is odd.

Definition 4.1. For an even (resp. odd) matchgate $\Gamma$ with arity $n$, the condensed signature $\left(g_{\alpha}\right)$ of $\Gamma$ is a vector of dimension $2^{n-1}$, and $g_{\alpha}=\Gamma^{\alpha b}$ (resp. $g_{\alpha}=\Gamma^{\alpha \bar{b}}$ ), where $\alpha \in\{0,1\}^{n-1}$ and $b=p(\alpha)$, and we say the condensed signature $g$ realized by the matchgate $\Gamma$

We prove the following theorem that characterizes the structure of blockwise symmetric matchgate signatures.

Theorem 4.1. Let $\Gamma=\left(\Gamma^{\alpha_{1} \alpha_{2} \cdots \alpha_{n}}\right)$ be a blockwise symmetric sigature with arity $n \ell$, where $n \geq 3$. If $\Gamma$ is realized by a matchgate, then there exists a condensed signature $\left(g_{\alpha}\right)_{\alpha \in\{0,1\}^{\ell}}$ that is realized by a matchgate with arity $\ell+1$, and a bitwise symmetric matchgate signature $\Gamma_{S}$ that is realized by a matchgate with arity $n$ such that

$$
\Gamma^{\alpha_{1} \alpha_{2} \cdots \alpha_{n}}=g_{\alpha_{1}} g_{\alpha_{2}} \cdots g_{\alpha_{n}} \Gamma_{S}^{p\left(\alpha_{1}\right) p\left(\alpha_{2}\right) \cdots p\left(\alpha_{n}\right)} .
$$

In [8], the following similar theorem was given.

Theorem 4.2. Let $\Gamma=\left(\Gamma^{\alpha_{1} \alpha_{2} \cdots \alpha_{n}}\right)$ be a blockwise symmetric signature with arity $n \ell$, where $n \geq 4$. If $\Gamma$ is realized by an even matchgate (resp. odd matchgate) and $\Gamma^{\mathbf{0 0} \cdots \mathbf{0}} \neq 0$ (resp. $\Gamma^{e_{1} \mathbf{0} \cdots \mathbf{0}} \neq 0$ ), then there exists a condensed signature $\left(g_{\alpha}\right)_{\alpha \in\{0,1\}^{\ell}}$ that is realized by a matchgate with arity $\ell+1$, and a bitwise symmetric matchgate signature $\Gamma_{S}$ that is realized by a matchgate with arity $n$ such that

$$
\Gamma^{\alpha_{1} \alpha_{2} \cdots \alpha_{n}}=g_{\alpha_{1}} g_{\alpha_{2}} \cdots g_{\alpha_{n}} \Gamma_{S}^{p\left(\alpha_{1}\right) p\left(\alpha_{2}\right) \cdots p\left(\alpha_{n}\right)} .
$$

Note that we improve Theorem 4.2 by removing the non-vanishing condition $\Gamma^{\mathbf{0 0} \cdots \mathbf{0}} \neq 0$ (or $\Gamma^{e_{\mathbf{1}} \mathbf{0} \cdots \mathbf{0}} \neq$ 0 ), and improving $n \geq 4$ to $n \geq 3$. Moreover, the counterexample given by $\Gamma_{1}$ in the end of Section 3 shows that the condition $n \geq 3$ is necessary.

For a blockwise symmetric matchgate signature $\Gamma=\left(\Gamma^{\alpha_{1} \alpha_{2} \cdots \alpha_{n}}\right)$ with arity $n \ell$, we have $\operatorname{rank}(M(\Gamma))=$ 0,1 or 2 by Lemma 3.1. If $\operatorname{rank}(M(\Gamma))=0$, the proof is trivial. In the following, we prove Theorem 4.1 for $\operatorname{rank}(M(\Gamma))=2$ and omit the proof for $\operatorname{rank}(M(\Gamma))=1$ due to the space limit. 
Proof. By Lemma 3.1, $M(\Gamma)$ has a full rank submatrix of the following form $\left(\begin{array}{cc}\Gamma^{\theta \gamma_{2} \cdots \gamma_{n}} \underset{0}{0} & 0 \\ 0 & \Gamma^{\eta\left(\gamma_{2}+e_{t}\right) \cdots \gamma_{n}}\end{array}\right)$ or

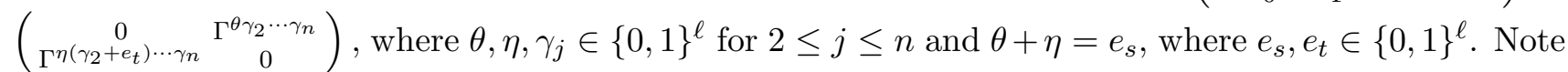
that $p(\theta) \neq p(\eta)$. Without loss of generality, we assume that $p(\theta)=0$ and $p(\eta)=1$ in the following.

Note that the rows $M(\Gamma)^{\theta}$ and $M(\Gamma)^{\eta}$ are linearly independent. Then by $\operatorname{rank}(M(\Gamma))=2$, all of the rows of $M(\Gamma)$ are linear combinations of $M(\Gamma)^{\theta}$ and $M(\Gamma)^{\eta}$. Moreover, for any $\alpha_{i}, \alpha_{j} \in\{0,1\}^{\ell}$, by the parity condition of $\Gamma$, if $p\left(\alpha_{i}\right) \neq p\left(\alpha_{j}\right)$, the nonzero entries of $M(\Gamma)^{\alpha_{i}}$ and $M(\Gamma)^{\alpha_{j}}$ are in disjoint column positions. So $M(\Gamma)^{\alpha_{i}}$ and $M(\Gamma)^{\alpha_{j}}$ are orthogonal. Thus for any $\alpha \in\{0,1\}^{\ell}$ there exists $g_{\alpha}$ such that $M(\Gamma)^{\alpha}=g_{\alpha} M(\Gamma)^{\theta}$ if $p(\alpha)=p(\theta)$, and $M(\Gamma)^{\alpha}=g_{\alpha} M(\Gamma)^{\eta}$ if $p(\alpha)=p(\eta)$.

Up to a global nonzero scalar, we can assume that $\Gamma^{\theta \gamma_{2} \cdots \gamma_{n}}=1$ and $\Gamma^{\eta\left(\gamma_{2}+e_{t}\right) \cdots \gamma_{n}}=r$, then for any $\alpha \in\{0,1\}^{\ell}, g_{\alpha}=\Gamma^{\alpha \gamma_{2} \cdots \gamma_{n}}$ if $p(\alpha)=p(\theta)$, and $g_{\alpha}=r^{-1} \Gamma^{\alpha\left(\gamma_{2}+e_{t}\right) \cdots \gamma_{n}}$ if $p(\alpha)=p(\eta)$. Now we prove that $g=\left(g_{\alpha}\right)$ is a condensed signature. Assume that $\Gamma=\left(\Gamma^{\alpha_{1} \alpha_{2} \cdots \alpha_{n}}\right)$ is realized by the matchgate $G$ with arity $n \ell$. Note that $G$ has $n \ell$ external nodes and we group the external nodes into $n$ blocks of consecutive $\ell$ nodes each. Firstly, we connect a path $L$ of length 2 to the $(\ell+t)$-th external node, i.e., the $t$-th node of the second block, of $G$. We denote the $(\ell+t)$-th external node of $G$, i.e., one endpoint of $L$, as $v_{1}$, another endpoint of $L$ as $v_{3}$ and the middle node of $L$ as $v_{2}$. If the $(\ell+t)$-th bit of $\theta \gamma_{2} \cdots \gamma_{n}$ is 0 , we give the edge between $v_{1}$ and $v_{2}$ weight $r^{-1}$ and the edge between $v_{2}$ and $v_{3}$ weight 1 . If the $(\ell+t)$-th bit of $\theta \gamma_{2} \cdots \gamma_{n}$ is 1 , we give the edge between $v_{1}$ and $v_{2}$ weight 1 and the edge between $v_{2}$ and $v_{3}$ weight $r^{-1}$, and view $v_{3}$ as an external node and $v_{1}, v_{2}$ as internal nodes. We do nothing to the external nodes in the first block and still view them as external nodes. We view other external nodes, i.e., the external nodes that are not in the first block and not the $(\ell+t)$-th external node, as internal node and do the following operations to them: for $\ell+1 \leq i \leq n \ell$ and $i \neq \ell+t$, if the $i$-th bit of $\theta \gamma_{2} \cdots \gamma_{n}$ is 0 , do nothing to $G$; if the $i$-th bit of $\theta \gamma_{2} \cdots \gamma_{n}$ is 1 , connect an edge with weight 1 to the $i$-th external node of $G$ and view the new nodes as internal nodes. Then we get a new matchgate $G^{\prime}$ with arity $\ell+1$ and $g$ is the condensed signature of $G^{\prime}$.

Now we construct the matchgate signature $\Gamma_{S}$ of arity $n$. For every block of $G$, for $1 \leq i \leq \ell$, if the $i$-th bit of $\theta$ is 1 then we add an edge of weight 1 to the $i$-th external node, and the new node replaces it as an external node. If the $i$-th bit of $\theta$ is 0 then we do nothing to it. We get a new matchgate $H$. Note that all the bits of $\theta, \eta$ are the same except the $s$-th bit. Next, we define $H^{\prime}$ from $H$ : In each block of $\ell$ external nodes of $H$, we pick only the $s$-th external node as an external node of $H^{\prime}$; all others are considered internal nodes of $H^{\prime}$. We denote the matchgate signature of $H^{\prime}$ by $\Gamma_{S}$. Then $\Gamma_{S}=\left(\Gamma_{S}^{j_{1} j_{2} \cdots j_{n}}\right)$ is a signature of arity $n$ and $\Gamma_{S}^{j_{1} j_{2} \cdots j_{n}}=\Gamma^{\alpha_{1} \alpha_{2} \cdots \alpha_{n}}$, where $\alpha_{i} \in\{\theta, \eta\}$ and $j_{i}=p\left(\alpha_{i}\right)$.

Now for any $\alpha_{1} \alpha_{2} \cdots \alpha_{n} \in\{0,1\}^{n \ell}$, we prove (4.1) by induction on $t$, the number of $\alpha_{i}$ from $\left\{\alpha_{1}, \alpha_{2}, \cdots, \alpha_{n}\right\}$ that do not belong to $\{\theta, \eta\}$ in $\left\{\alpha_{1}, \alpha_{2}, \ldots, \alpha_{n}\right\}$. Note that $g_{\theta}=g_{\eta}=1$. If all $\alpha_{i}$ belong to $\{\theta, \eta\}$, i.e., $t=0$, we are done by the definition of $\Gamma_{S}^{j_{1} j_{2} \cdots j_{n}}$ and (4.1) is proved. Inductively we assume that (4.1) has been proved for $t-1$ and there are $t \geq 1$ blocks in $\left\{\alpha_{1}, \alpha_{2}, \ldots, \alpha_{n}\right\}$ that that do not belong to $\{\theta, \eta\}$. Then we pick one $\alpha_{i}$ that do not belong to $\{\theta, \eta\}$. If $\alpha_{i}$ has the same parity as $\theta$, then the $\alpha_{i}$-th row $M(\Gamma)^{\alpha_{i}}=g_{\alpha_{i}} M(\Gamma)^{\theta}$ in $M(\Gamma)=\left(\begin{array}{cccc}\cdots & \Gamma^{\theta \alpha_{1} \cdots \alpha_{i-1} \alpha_{i+1} \cdots \alpha_{n}} & \cdots \\ \cdots & \ldots \\ \cdots & \Gamma^{\alpha_{i} \alpha_{1} \cdots \alpha_{i-1} \alpha_{i+1} \cdots \alpha_{n}} & \cdots \\ \cdots & & \cdots & \cdots\end{array}\right)$. Thus $\Gamma^{\alpha_{1} \alpha_{2} \cdots \alpha_{n}}=\Gamma^{\alpha_{i} \alpha_{1} \cdots \alpha_{i-1} \alpha_{i+1} \cdots \alpha_{n}}=$ $g_{\alpha_{i}} \Gamma^{\theta \alpha_{1} \cdots \alpha_{i-1} \alpha_{i+1} \cdots \alpha_{n}}$. Note that the number of blocks from $\left\{\theta, \alpha_{1}, \cdots, \alpha_{i-1}, \alpha_{i+1}, \cdots, \alpha_{n}\right\}$ that do not belong to $\{\theta, \eta\}$ is $t-1$. Thus we have $\Gamma^{\theta \alpha_{1} \cdots \alpha_{i-1} \alpha_{i+1} \cdots \alpha_{n}}=g_{\alpha_{2}} \cdots g_{\alpha_{i-1}} g_{\alpha_{i+1}} \cdots g_{\alpha_{n}} \Gamma_{S}^{p(\theta) p\left(\alpha_{2}\right) \cdots p\left(\alpha_{n}\right)}$ by induction. Then $\Gamma^{\alpha_{1} \alpha_{2} \cdots \alpha_{n}}=g_{\alpha_{1}} g_{\alpha_{2}} \cdots g_{\alpha_{n}} \Gamma_{S}^{p\left(\alpha_{1}\right) p\left(\alpha_{2}\right) \cdots p\left(\alpha_{n}\right)}$ and we complete the proof. If $p\left(\alpha_{i}\right)=p(\eta)$, the proof is similar and we omit it here. 


\section{References}

[1] Jin-Yi Cai, Zhiguo Fu: A collapse theorem for holographic algorithms with matchgates on domain size at most 4. Inf. Comput. 239: 149-169 (2014).

[2] Jin-Yi Cai, Zhiguo Fu: Holographic Algorithm with Matchgates Is Universal for Planar \#CSP Over Boolean Domain. To appear at STOC 2017. The full version is available at https://arxiv.org/abs/1603.07046.

[3] Jin-Yi Cai, Zhiguo Fu, Heng Guo, Tyson Williams: A Holant Dichotomy: Is the FKT Algorithm Universal? FOCS 2015: 1259-1276. The full version is availabel at https://arxiv.org/abs/1505.02993.

[4] Jin-Yi Cai, Aaron Gorenstein: Matchgates Revisited. Theory of Computing 10: 167-197 (2014).

[5] Jin-Yi Cai, Heng Guo, Tyson Williams: A Complete Dichotomy Rises from the Capture of Vanishing Signatures: extended abstract. STOC 2013: 635-644. The full version is available at http://arxiv.org/abs/1204.6445.

[6] Jin-Yi Cai, Pinyan Lu: Holographic algorithms: from art to science. STOC 2007: 401-410.

[7] Jin-yi Cai, Pinyan Lu: Holographic algorithms: The power of dimensionality resolved. Theor. Comput. Sci. 410(18): 1618-1628 (2009).

[8] Jin-Yi Cai, Pinyan Lu: On blockwise symmetric signatures for matchgates, Theoretical Computer Science 411 (2010) 739-750.

[9] Jin-Yi Cai, Pinyan Lu, Mingji Xia: Holant problems and counting CSP. STOC 2009: 715-724.

[10] Jin-Yi Cai, Pinyan Lu, Mingji Xia: Holographic Algorithms with Matchgates Capture Precisely Tractable Planar \#CSP. FOCS 2010: 427-436.

[11] Sitan Chen: Basis collapse for holographic algorithms over all domain sizes. STOC 2016: 776-789.

[12] N. Creignou and M. Hermann, Complexity of generalized satisfiability counting problems, Information and Computation 125 (1996), 112.

[13] Heng Guo, Sangxia Huang, Pinyan Lu, Mingji Xia: The Complexity of Weighted Boolean \#CSP Modulo k. STACS 2011: 249-260.

[14] Heng Guo and Tyson Williams: The Complexity of Planar Boolean \#CSP with Complex Weights. ICALP (1) 2013: 516-527.

[15] Heng Guo, Pinyan Lu, Leslie G. Valiant: The Complexity of Symmetric Boolean Parity Holant Problems. SIAM J. Comput. 42(1): 324-356 (2013)

[16] Sangxia Huang, Pinyan Lu: A Dichotomy for Real Weighted Holant Problems. IEEE Conference on Computational Complexity 2012: 96-106.

[17] P. W. Kasteleyn, The Statistics of Dimers on a Lattice, Physica 27 (1961) 1209-1225.

[18] P. W. Kasteleyn, Graph Theory and Crystal Physics. In Graph Theory and Theoretical Physics, (F. Harary, ed.), Academic Press, London, 43-110 (1967).

[19] J. M. Landsberg, Jason Morton, Serguei Norine: Holographic algorithms without matchgates. CoRR abs/0904.0471 (2009)

[20] H. N. V. Temperley and M. E. Fisher. Dimer Problem in Statistical Mechanics - an Exact Result. Philosophical Magazine 6: 1061- 1063 (1961).

[21] Leslie G. Valiant, The Complexity of Enumeration and Reliability Problems, SIAM J. Comput., 8(3)(1979), 410-421.

[22] Leslie G. Valiant: Quantum Circuits That Can Be Simulated Classically in Polynomial Time. SIAM J. Comput. 31(4): 1229-1254 (2002)

[23] Leslie G. Valiant: Expressiveness of matchgates. Theor. Comput. Sci. 289(1): 457-471 (2002). 
[24] Leslie G. Valiant: Accidental algorithms, Proc. 47th Annual IEEE Symposium on Foundations of Computer Science, Oct 22 -24, Berkeley, CA, IEEE Press, (2006), 509-517.

[25] Leslie. G. Valiant. Holographic Algorithms, SIAM J. Comput., 37(5) (2008) 1565-1594. A preliminary version appeared in FOCS 2004: 306-315.

[26] Leslie G. Valiant: The complexity of computing the permanent. Theoretical Computer Science, 8 (1979), pp. 189-201.

[27] L. G. Valiant. Some observations on holographic algorithms, Proc. 9th Latin American Theoretical Informatics Symposium, LATIN 2010. LNCS, Vol 6034 Springer-Verlag (2010), 577-590.

[28] Mingji Xia: Base collapse of holographic algorithms. STOC 2016: 790-799. 